\title{
Universiteit
}

Leiden

The Netherlands

\section{Brownian fluctuations and heating of an optically aligned gold nanorod} Ruijgrok, P.V.; Verhart, N.R.; Zijlstra, P.; Tchebotareva, A.L.; Orrit, M.A.G.J.

\section{Citation}

Ruijgrok, P. V., Verhart, N. R., Zijlstra, P., Tchebotareva, A. L., \& Orrit, M. A. G. J. (2011). Brownian fluctuations and heating of an optically aligned gold nanorod. Physical Review Letters, 107(3), 037401. doi:10.1103/PhysRevLett.107.037401

Version: $\quad$ Not Applicable (or Unknown)

License: $\quad$ Leiden University Non-exclusive license

Downloaded from: https://hdl.handle.net/1887/61327

Note: To cite this publication please use the final published version (if applicable). 


\title{
Brownian Fluctuations and Heating of an Optically Aligned Gold Nanorod
}

\author{
P. V. Ruijgrok, ${ }^{1}$ N. R. Verhart, ${ }^{1}$ P. Zijlstra, ${ }^{1}$ A. L. Tchebotareva, ${ }^{1}$ and M. Orrit ${ }^{1}$ \\ ${ }^{1}$ Institute of Physics, Leiden University, P.O. Box 9504, 2300 RA Leiden, The Netherlands
}

(Received 20 December 2010; published 11 July 2011)

\begin{abstract}
We present the first quantitative measurements of the torque exerted on a single gold nanorod in a polarized three-dimensional optical trap. We determined the torque both by observing the time-averaged orientation distribution and by measuring the dynamics of the rotational Brownian fluctuations. The measurements are in good agreement with calculations, where the temperature profile around the hot nanorod gives rise to a reduced, effective viscosity. The maximum torque on a $60 \mathrm{~nm} \times 25 \mathrm{~nm}$ nanorod was $100 \mathrm{pN} \cdot \mathrm{nm}$, large enough to address single-molecule processes in soft and biological matter.
\end{abstract}

Since their invention some 30 years ago [1,2], optical tweezers have become a versatile tool to study the mechanics of soft matter, investigate the statistical mechanics of model systems, and enable fabrication on the nanometer scale [3]. Rapid advances in optical trapping techniques have led to new methods by which both forces and torques can be exerted and measured. Most of those exploit the action of a polarized trap laser on an optically anisotropic microparticle [4,5]. These advances have led to a better understanding of the rotational Brownian motion of a microparticle in the laser potential [6], and resulted in the first simultaneous measurement of torque, angle, force and position during supercoiling of DNA [7].

Applications in environments that are structured on the nanometer scale, as found in soft matter systems, require trapping handles smaller than-or comparable in size tothe structures under study. However, optical trapping of dielectric particles below $300 \mathrm{~nm}$ in diameter is difficult. In contrast, the large polarizability of a metal compared to a dielectric enables the stable trapping of spherical gold nanoparticles [8] down to a diameter of $9.5 \mathrm{~nm}$ [9]. The optical forces that can be exerted on a metal nanoparticle have been characterized for gold and silver nanospheres [10-12] and gold nanorods [13]. The shape and volume of the metal nanoparticles largely determine the accessible forces, which range from 0.1 to $10 \mathrm{pN}$ for gold nanorods smaller than $100 \mathrm{~nm}$, i.e., relevant forces for many biophysical and soft matter systems [14]. More interestingly still, nonspherical metal nanoparticles also experience torques in optical traps, because of the anisotropy of their polarizability tensor $[15,16]$. Simultaneously applying a torque and a force to a metal nanoparticle would be particularly appealing for single-molecule experiments. However, no quantitative measurements of optical torques on such small particles have been published yet.

In this Letter, we demonstrate that a single gold nanorod of $25 \mathrm{~nm}$ in diameter and $60 \mathrm{~nm}$ in length can be used to exert optical torques of up to $100 \mathrm{pN} \cdot \mathrm{nm}$ in a linearly polarized, three-dimensional optical trap. The restoring optical torque leads to a strong alignment of the rod, limiting the amplitude of its Brownian orientation fluctuations to about 14 degrees. In addition, we exploit the dipolar character of the rod's longitudinal plasmon resonance [17] to accurately determine the optical torque in two independent ways: (1) via the time-averaged orientation distribution deduced from white-light scattering spectra, and (2) through a dynamical analysis of rotational Brownian fluctuations observed in the polarized scattered intensity. We determine the rod's heating by the trap beam and provide the first data on the combined translational and rotational Brownian motions of a hot object in an optical trap.

The optical setup is schematically shown in Fig. 1(a) and consists of a single beam optical trap at $1064 \mathrm{~nm}(5 \mathrm{~W}$ IPG Photonics Ytterbium fiber laser). The trapping beam is focused into water about $25 \mu \mathrm{m}$ away from the glass substrate by an objective with a high numerical aperture (Olympus oil immersion $60 \times, 1.4 \mathrm{NA}$ ). To alleviate the spherical aberrations introduced by water's too low index, the incoming trapping beam was made slightly convergent

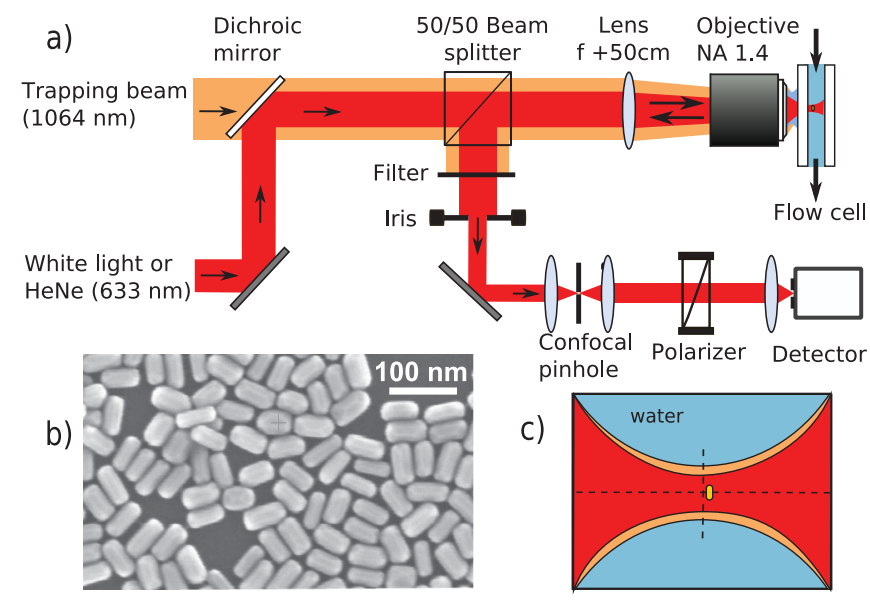

FIG. 1 (color). (a) Scheme of the optical trap. (b) Scanning electron micrograph of some gold nanorods. (c) Scheme of a rod trapped in the laser focus in water. The rod is slightly shifted along the axis by radiation pressure. 
by means of a $50 \mathrm{~cm}$ lens just before the objective [18]. We estimated an effective numerical aperture of 1.0 at the trap focus [19]. To obtain scattering spectra, white light from a xenon arc lamp was focused to a spot and overlapped with the trap focus, see Fig. 1(c). Scattered light was collected by the focusing objective and detected by a nitrogencooled CCD camera coupled to a spectrograph. An iris inserted into the detection path selected the center $5 \mathrm{~mm}$ of the beam, to minimize depolarization effects by the objective [19]. We inserted a $50 \mu \mathrm{m}$ confocal pinhole at the focus of a $10 \mathrm{~cm}$ lens to reduce the background. Translation and rotation dynamics of the trapped rods were deduced from the backscattered light of a HeNe laser $(633 \mathrm{~nm})$, detected by a single-photon counting photodiode and analyzed with a correlation card. Gold nanorods with $60 \mathrm{~nm}$ average length and $25 \mathrm{~nm}$ average diameter, shown in the electron micrograph in Fig. 1(b), were synthesized by the silver-assisted seed-mediated method [20] and coated with thiolated polyethyleneglycol (mPEG, MW5000, Sigma Aldrich) to prevent their aggregation in pure water [21]. The rod suspensions were diluted with ultrapure water to limit the trapping of multiple particles during the course of a measurement, up to 2 hours.

White-light scattering spectra of a trapped nanorod are shown in Fig. 2(a). The parallel spectrum (analyzer along the trap polarization), displays the strong longitudinal plasmon, with the maximum at $625 \mathrm{~nm}$. The (nearly) Lorentzian shape of the spectrum and its narrow width (49 nm FWHM) confirm that only a single particle is trapped [19]. With an analyzer perpendicular to the trap's polarization, the scattered signal is much weaker. Its spectrum shows residual intensity from the longitudinal plasmon at $625 \mathrm{~nm}$ and the transverse plasmon at about $550 \mathrm{~nm}$.

Because of the dipolar angular dependence of the scattered intensity, the time-averaged intensity $\left\langle I_{\perp}\right\rangle_{t}$ of the longitudinal plasmon observed in the perpendicular direction mainly arises from small angular fluctuations of the rod around its equilibrium orientation along the trap polarization [19]. The intensity ratio $\left\langle I_{\|}\right\rangle_{t} /\left\langle I_{\perp}\right\rangle_{t}$ can be directly related to the ratio of the rotational trapping energy (or trap depth) to the thermal energy $k_{B} T$. The timeaveraged intensities of the longitudinal plasmon in the two directions are the thermal expectation values $I_{0}\left\langle\cos ^{2} \theta\right\rangle_{T}$ and $I_{0}\left\langle\sin ^{2} \theta \cos ^{2} \phi\right\rangle_{T}(\theta$ and $\phi$ are the polar angles of the rod axis [19]). The probability of finding angles $\theta, \phi$ is given by a Boltzmann distribution $\exp \left\{-U(\theta) / k_{B} \tilde{T}_{B}\right\}$, with an effective temperature $\tilde{T}_{B}$ accounting for heating by the absorbed trap light. The potential energy $U$ is that of the rod's induced dipole in the optical field $E_{0}$, given by $U(\theta)=-\frac{1}{4} \operatorname{Re}\{\Delta \alpha\} E_{0}^{2} \cos ^{2} \theta=-\frac{1}{2} \kappa_{r} \cos ^{2} \theta . \quad \operatorname{Re}\{\Delta \alpha\}=$ $\operatorname{Re}\left\{\alpha_{L}-\alpha_{T}\right\}$ is the difference between the real parts of the longitudinal and transverse polarizabilities of the nanorod at the trap wavelength, and $\kappa_{r}$ is the rotational trap stiffness, equal to the rotational spring constant of the trap for small angles. In the limit of high rotational trap
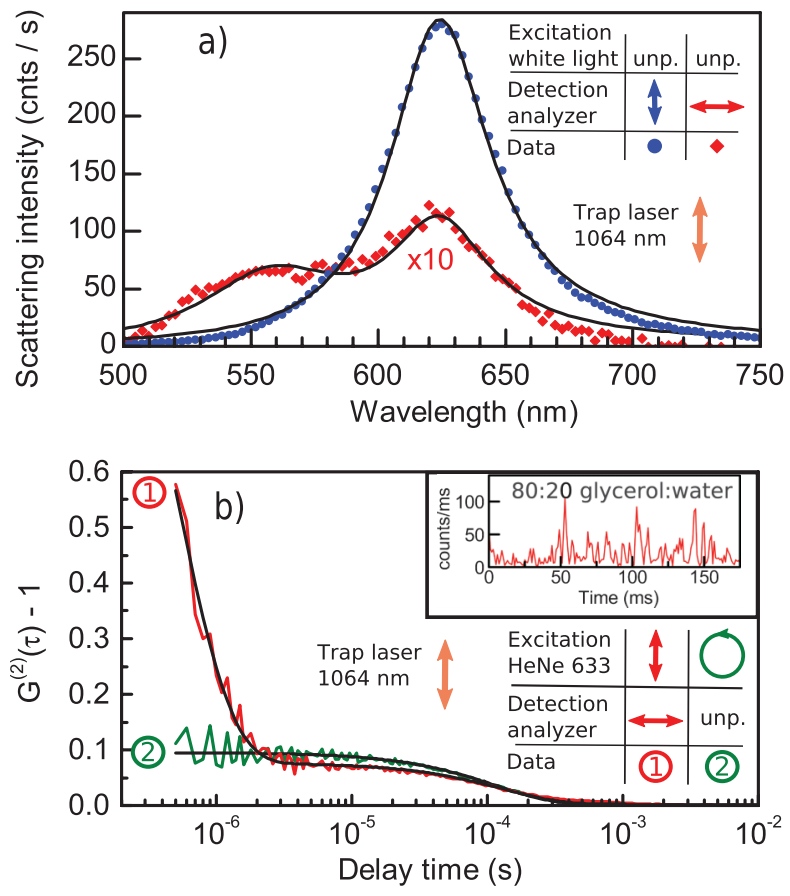

FIG. 2 (color). (a) Scattering spectra of an optically trapped gold nanorod in water, recorded with unpolarized excitation and analyzed parallel (blue circles) or perpendicular (red diamonds) to the trap polarization. The solid lines are Lorentzian fits (in frequency). Trapping power $80 \mathrm{~mW}$ at the focal plane, integration time 15 s. (b) Autocorrelation functions of light from a HeNe laser, scattered by a trapped nanorod in water. Data set 1 (Red): Same nanorod as (a), with an analyzer in the perpendicular direction. Data set 2 (Green): A different nanorod than (a), with circularly polarized excitation and without analyzer in the detection. The solid curves are (bi-)exponential fits (characteristic times $0.48 \pm 0.01 \mu \mathrm{s}$ and $132 \pm 8 \mu \mathrm{s}$ for data set 1 , and $96 \pm 2 \mu$ s for data set 2 ). Trapping power $80 \mathrm{~mW}$ at the focal plane, acquisition time $20 \mathrm{~s}$. Inset: Time trace of the intensity scattered by a rod trapped in a glycerol-water mixture, directly showing slowed down orientation fluctuations. Photon counts have been grouped in $1 \mathrm{~ms}$ time bins.

stiffness, the spectral intensity ratio is well approximated by $\left\langle I_{\|}\right\rangle_{t} /\left\langle I_{\perp}\right\rangle_{t} \simeq \kappa_{r} /\left(k_{B} \tilde{T}_{B}\right)-3$ [19]. An intensity ratio $\left\langle I_{\|}\right\rangle_{t} /\left\langle I_{\perp}\right\rangle_{t}=29$ determined from the data in Fig. 1(a) thus directly yields a trap depth $\kappa_{r} / 2$ of $16 k_{B} \tilde{T}_{B}$. This corresponds to root-mean-square angular fluctuations $\theta_{\mathrm{RMS}} \approx \sqrt{2 k_{B} \tilde{T}_{B} / \kappa_{r}}=14^{\circ}$. The maximum torque that can be exerted is $\kappa_{r} / 2 \approx 100 \mathrm{pN} \cdot \mathrm{nm}$.

The angular trapping of the rod can also be characterized by the dynamics of the rotational Brownian motion. We excited the rod with a $\mathrm{HeNe}$ laser at $633 \mathrm{~nm}$ linearly polarized along the trap polarization, and detected the scattered light behind an analyzer oriented in the perpendicular direction, see Fig. 2(b), curve 1. As the rod wiggles around its equilibrium, the detected signal fluctuates between zero and positive values, as scattering from the transverse band and residual background are negligible. 
This signal is displayed in the inset of Fig. 2(b) for a nanorod trapped in a viscous water-glycerol mixture, where the rotational dynamics are slowed down. Figure 2(b) shows the normalized autocorrelation function of the light scattered by the gold nanorod of Fig. 2(a), trapped in water. The correlation function is well fitted by a bi-exponential decay. We attribute the fast relaxation to rotational fluctuations of the rod in the trap. The slower relaxation is due to the transverse and axial translations of the rod in the focus, which also modulate the scattered intensity. To confirm this attribution, we recorded autocorrelation functions with circularly polarized excitation and unpolarized detection, shown in Fig. 2(b), data set 2. These measurements indeed present only the slow translational part, without the fast relaxation due to orientation fluctuations. In the limit of strong alignment, the rotational correlation time $\tau_{r}$ is half the macroscopic relaxation time of the rod in the trap, itself the ratio of the rotational friction coefficient $\xi_{r}$ to the trap stiffness $\kappa_{r}$ [19]: $\tau_{r}=\xi_{r} / 2 \kappa_{r}$, where $\xi_{r}=\eta V C_{r}, \eta$ is the viscosity of water, $V$ the hydrodynamic volume, and $C_{r}$ a geometrical factor.

We now correlate the time-averaged and dynamic measurements on the same particle. Whereas the timeaveraged spectral ratio directly measures the competition between the trap energy and the thermal fluctuations, the rotational correlation time probes the temperaturedependent viscosity. Figures 3(a) and 3(b) show these two quantities for the same nanorod, versus trapping power. The plot of the intensity ratio $\left\langle I_{\|}\right\rangle_{t} /\left\langle I_{\perp}\right\rangle_{t}$ versus trap intensity in Fig. 3(a) shows a distinct downward curvature, which we attribute to an increase of local temperature with trap power. Indeed, the optical restoring torque on the nanorod competes with stronger thermal fluctuations as the rod gets hotter. Similarly, the plots of the inverse rotational and translational times $1 / \tau_{r}$ and $1 / \tau_{t}$ in Figs. 3(b) and 3(c) show an upward curvature because of the reduced water viscosity at higher trap powers.

The Brownian motion of the hot nanorod takes place in an inhomogeneous temperature and viscosity profile. Heat diffusion being much faster than molecular diffusion, the temperature profile accompanies the particle in its motion [22]. We describe these fluctuations phenomenologically by means of an effective temperature and of effective viscosities, as proposed recently for translational diffusion of a hot free particle [22]. This hot Brownian motion (HBM) has an effective temperature $T_{\mathrm{HBM}}$ close to the average $\left(T_{p}+T_{0}\right) / 2$ between the particle temperature $T_{p}$ and the bath temperature $T_{0}$, and an effective viscosity $\eta_{\text {HBM }}$. Here, we introduce another effective viscosity for the rotational HBM. This new parameter is needed because the plot of the rotational inverse time versus the translational inverse time [see Fig. 3(d)] is strongly nonlinear, indicating that these two effective viscosities must be different (see further data from other rods [19]). Indeed, taking the same effective viscosity for both translations and
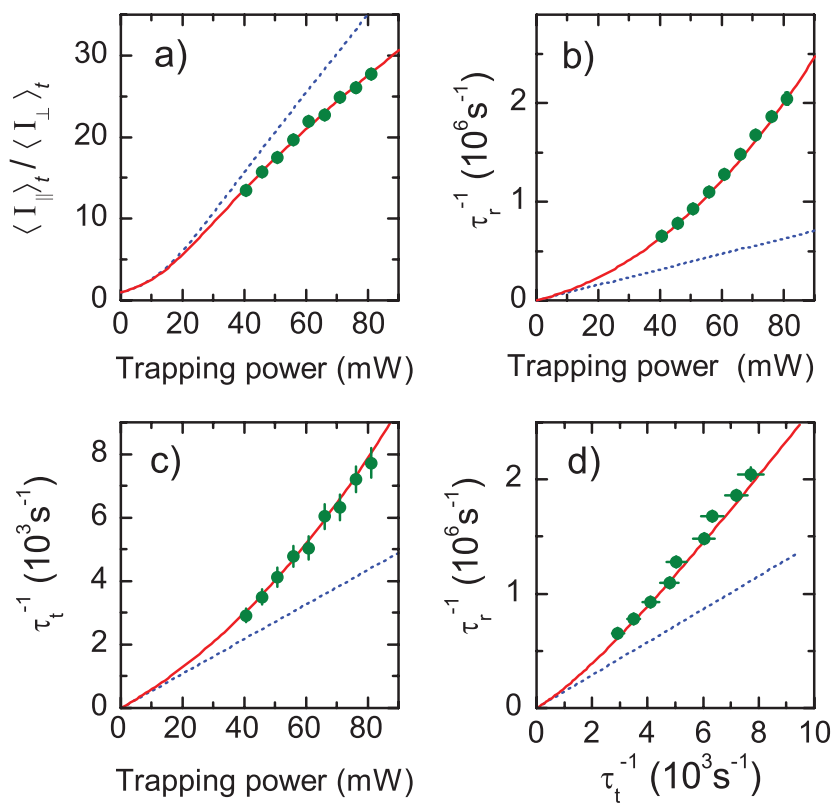

FIG. 3 (color). Trap characteristics as functions of trapping power (at the focal plane), for the nanorod of Fig. 2. (a) Ratio of scattered intensities of the rod's longitudinal plasmon resonance, parallel and perpendicular to the trapping laser polarization. (b) Inverse of the rotation correlation time $\tau_{r}$. (c) Inverse of the translation correlation time $\tau_{t}$. (d) Inverse rotation correlation time versus inverse translation correlation time. The red lines show a global fit with an effective temperature $\tilde{T}_{B}$ deduced from (a) and effective viscosities for translations $\tilde{\eta}_{t}$ from (c) and rotations $\tilde{\eta}_{r}$ from (b). The blue dotted lines would be observed in the complete absence of heating. In (d), the blue dotted line follows from taking the same effective viscosity for rotation and translation, $\tilde{\eta}_{r}=\tilde{\eta}_{t}$.

rotations would lead to the straight dotted line in Fig. 3(d), with slope $B \kappa_{r} / \kappa_{t}$ (B depending only on the shape and size of the particle under study).

To find the effective temperatures and viscosities, we globally fitted the data of Fig. 3 and those of two other particles measured under the same conditions to a model [19] involving an effective temperature $\tilde{T}_{B}$ and two effective viscosities $\tilde{\eta}_{r}$ and $\tilde{\eta}_{t}$. We adjusted the unknown parameters in the following way. The local trap intensity was obtained from the spectral intensity ratio and from the polarizability $\operatorname{Re}\{\Delta \alpha\}$ of the three nanorods. That polarizability was calculated in the electrostatic approximation, with appropriate corrections to account for radiation damping and electron-surface scattering $[17,19,23]$. The aspect ratio of the ellipsoid and the plasmon damping rate were adjusted for each rod to reproduce the resonance wavelength and width measured in scattering spectra [19]. The intensity in the trap and the average volume of the 3 measured rods were jointly fitted to remain compatible with the volume distribution obtained from electron microscopy.

With this local intensity and the rotational times, we adjusted the rods' hydrodynamic volumes. We calculated 
friction coefficients from Perrin's expressions for a prolate spheroid translated along the principal axes or rotated around a short principal axis [24]. The fitted effective hydrodynamic volume accounts for the mPEG layer (effective thickness $5 \mathrm{~nm}[25]$ ). The effective temperature $\tilde{T}_{B}$ was fitted by the intensity ratios and found to be close to the particle's temperature $T_{p}$ calculated independently from the trap intensity. The effective translational viscosity was taken as $\eta_{\mathrm{HBM}}$, whereas the rotational effective viscosity was varied to fit the temperature dependence of rotational times in Fig. 3. The viscosity $\tilde{\eta}_{r}$ found was close to its minimum possible value, water's viscosity at the particle's temperature. The trap intensity found at the nanorod position was about $50 \%$ of the value expected for the focus of a diffraction-limited spot with the effective numerical aperture of 1.0 and the measured objective transmission of $19 \%$. We attribute this low value to residual spherical aberrations.

It is reasonable that the effective temperature $\tilde{T}_{B}$ of the orientation distribution should be close to the particle's temperature $T_{p}$. Indeed, the particle should be in thermal quasiequilibrium with the liquid shell around it. The effective translational viscosity $\tilde{\eta}_{t}$ should be close to that of the HBM, because our fat rod closely resembles a sphere. The difference in effective viscosities for the rotational and translational Brownian motions, however, is a surprise. Although rotations occur on much shorter times (microseconds) than translations (milliseconds), inertial effects appear too weak to explain this difference [19]. We note that the amplitude of the rotational motion, less than $10 \mathrm{~nm}$ for the tip in a rotation of 20 degrees, is much smaller than the amplitude of the translational motion. The difference in viscosities may thus arise from the PEG brush, which must follow the rod's translation but not necessarily its rotation.

Our model yields a temperature increase of the rod in the trap of about $70 \mathrm{~K}$ at the highest trapping power of $80 \mathrm{~mW}$, or about $0.9 \mathrm{~K} / \mathrm{mW}$. This value is fairly well reproduced by a simple model taking into account the fitted trap laser intensity, the calculated absorption cross section (electrostatic approximation), and heat dissipation [19]. Similar heating rates were previously found for gold spheres $[11,26]$. Such temperature rises may be of concern for certain applications. However, the temperature around the nanorod decays rapidly with distance, with a characteristic length of the order of the particle's radius. At a distance $100 \mathrm{~nm}$ away from the rod, the temperature increase is only about 10 degrees [19] for the highest trapping power used here.

We have determined for the first time the torque that can be exerted on a single gold nanorod in a polarized optical trap. The maximum torque we measured was $100 \mathrm{pN} \cdot \mathrm{nm}$, large enough to address typical single-molecule processes [7]. The small volume of a single gold nanorod compared to conventional dielectric particles paves the way to study environments which exhibit mechanical heterogeneity on nanometer length scales.

We thank Dr. A. Gaiduk for help with software, and Ziyu Gu for synthesis. Support and advice by Prof. L. B. Oddershede is gratefully acknowledged. This work was funded by the Foundation for Fundamental Research on Matter (FOM). A. T. and P.Z. acknowledge support from the Dutch Organization for Scientific Research (NWO) and by the ERC (Advanced Grant SiMoSoMa).

[1] A. Ashkin, Science 210, 1081 (1980).

[2] A. Ashkin, J. M. Dziedzic, J. E. Bjorkholm, and S. Chu, Opt. Lett. 11, 288 (1986).

[3] D. G. Grier, Nature (London) 424, 810 (2003).

[4] M. E. J. Friese, T. A. Nieminen, N. R. Heckenberg, and H. Rubinsztein-Dunlop, Nature (London) 394, 348 (1998).

[5] A. I. Bishop, T. A. Nieminen, N. R. Heckenberg, and H. Rubinsztein-Dunlop, Phys. Rev. Lett. 92, 198184 (2004).

[6] A. La Porta and M. D. Wang, Phys. Rev. Lett. 92, 190801 (2004).

[7] C. Deufel et al., Nat. Methods 4, 223 (2007).

[8] K. Svoboda and S. M. Block, Opt. Lett. 19, 930 (1994).

[9] F. Hajizadeh and S. N.S. Reihani, Opt. Express 18, 551 (2010).

[10] P. M. Hansen, N. Harrit, and L. Oddershede, Nano Lett. 5, 1937 (2005).

[11] Y. Seol, A. E. Carpenter, and T. T. Perkins, Opt. Lett. 31, 2429 (2006)

[12] L. Bosanac, T. Aabo, P. Bendix, and L. Oddershede, Nano Lett. 8, 1486 (2008).

[13] C. Selhuber-Unkel et al., Nano Lett. 8, 2998 (2008).

[14] K. Neuman and A. Nagy, Nat. Methods 5, 491 (2008).

[15] M. Pelton et al., Opt. Lett. 31, 2075 (2006).

[16] L. Tong, V. D. Miljković, and M. Käll, Nano Lett. 10, 268 (2010).

[17] C. Sönnichsen et al., Phys. Rev. Lett. 88, 077402 (2002).

[18] S. N. S. Reihani, M. A. Charsooghi, H. R. Khalesifard, and R. Golestian, Opt. Lett. 31, 766 (2006).

[19] See supplemental material at http://link.aps.org/ supplemental/10.1103/PhysRevLett.107.037401 for details of experimental methods, scattering spectra of multiple trapped gold nanorods, a study of the reshaping of rods in the trap, trap characteristics as a function of trapping power for two additional gold nanorods and a description of the model used to find the effective temperatures and viscosities.

[20] B. Nikoobakht and M. A. El-Sayed, Chem. Mater. 15, 1957 (2003).

[21] C. Fernández-López et al., Langmuir 25, 13894 (2009).

[22] D. Rings et al., Phys. Rev. Lett. 105, 090604 (2010).

[23] C. Novo et al., Phys. Chem. Chem. Phys. 8, 3540 (2006).

[24] F. Perrin, J. Phys. Radium 5, 497 (1934).

[25] B. M. I. van der Zande, J. K. G. Dhont, M. R. Böhmer, and A. P. Philipse, Langmuir 16, 459 (2000).

[26] P. M. Bendix, S. N. S. Reihani, and L. B. Oddershede, ACS Nano 4, 2256 (2010). 